\title{
The da Vinci Surgical System versus the Radius Surgical System
}

\author{
Norihiko Ishikawa*, Go Watanabe, Noriyuki Inaki, Hideki Moriyama, Masanari Shimada, \\ Masahiko Kawaguchi \\ Department of General and Cardiothoracic Surgery, Kanazawa University, Kanazawa, Japan \\ Email: *iskwnrhk@gmail.com
}

Received May 1, 2012; revised June 12, 2012; accepted June 27, 2012

\begin{abstract}
Objective: Kanazawa University introduced the da Vinci surgical system and the Radius surgical system. In this study, we compared the advantages and disadvantages of each system. Methods: The da Vinci system is a master-slave telemanipulation system, which provides hi-resolution 3D images. The Radius system is pair of hand-guided surgical manipulators. In this study we focus on the operability of both instruments rather than their 3D optical systems. Results: The Radius was originally developed specifically focused on ligation and suturing with suture sizes bigger than 4-0, it is more effective, less expensive compared with the da Vinci. Although the da Vinci system is bulky, it allows surgeons to perform endoscopic surgeries only if ports are properly placed to prevent each arm from colliding with the other arms. A crucial difference between the Radius and the da Vinci is not limited to anastomose small vessels but is extended to multidirectional dissection. Currently, the cost including initial investment is the biggest issue; however, the da Vinci is absolutely necessary to implement delicate cardiac surgeries endoscopically and less-invasively. Early approval of robotic surgery by the government is urgently required in Japan. Conclusions: Although both the da Vinci and the Radius have endoscopic instruments with a multi-degree of freedom, applications need to be differentiated depending on the procedures and indications. Therefore, it can be clearly said that these unique innovative systems will never compete against each other.
\end{abstract}

Keywords: da Vinci Surgical System; Radius Surgical System

\section{Introduction}

Kanazawa University introduced the da Vinci surgical system (Intuitive Surgical Inc., Sunnyvale, CA) in 2005 and the Radius surgical system (Tuebingen Scientific Medical GmbH, Germany) in 2006 [1-3]. The university is the only facility in Japan in which both innovative endoscopic surgery systems are in use. While surgeons unfamiliar with both systems may feel that the da Vinci is unnecessary if the Radius system is already being employed, this facility has benefited from the use of both innovative endoscopic surgery systems in Japan. We have therefore compared the advantages and disadvantages of each system.

\section{Comparison of the da Vinci and the Radius Surgical Systems}

The da Vinci system consists of an ergonomically designed surgeon's console, a patient-side cart with three or four interactive robotic arms and a Vision System. The da Vinci is a master-slave telemanipulation system that

"Corresponding author. provides 3D images with superior resolution and high contrast through its optical system (Figure 1(A)). The surgeon manipulates two master controllers located at the surgeon's console as in open surgery, and is able to obtain 3D images of the operative field, including binocular and magnified views with resolution equivalent to images obtained in an open fashion [4,5]. The Radius system consists of two hand-guided surgical manipulators, each approximately $50 \mathrm{~cm}$ in length, which is slightly longer than standard endoscopic instruments (Figure 1(B)) $[3,6,7]$. In this study we focus on the operability of both instruments rather than their 3D optical systems.

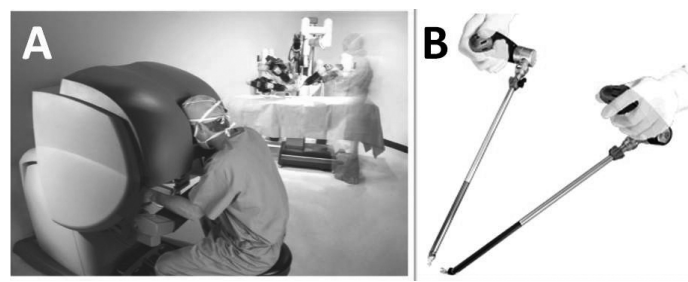

Figure 1. da Vinci surgical system (A) and radius surgical system (B). 


\subsection{Freedom of Instruments}

The da Vinci's instruments are designed to have 7 degrees of freedom and 90 degrees of articulation, allowing each instrument to function as if it had a human wrist. The Radius has a deflectable and rotatable tip that provides 7 degrees of handling freedom (Figure 2(A)). However, one of the Radius's finger tips are fixed and cannot move and its "wrist" has limited articulation (Figure 2(B)). Both systems seem to have 7 degrees of freedom; however, the da Vinci's instrument tips have complete freedom and articulation rather than the Radius'.

\subsection{Instrument Tips}

While the da Vinci has more than 40 instrument tips, including electric cauteries and an ultrasonic coagulating device and the entire instrument is changed when tips are changed, the Radius has interchangeable surgical endoeffectors with only 4 tips and no electric cautery (Figure 3). Because the Radius does not have a cautery tip, it can only be used for suturing and tying knots but not for tissue dissection. For both systems, changing instruments can be done intra-operatively, which requires only a few seconds.

\subsection{Dexterity}

The da Vinci system seamlessly translates the move-
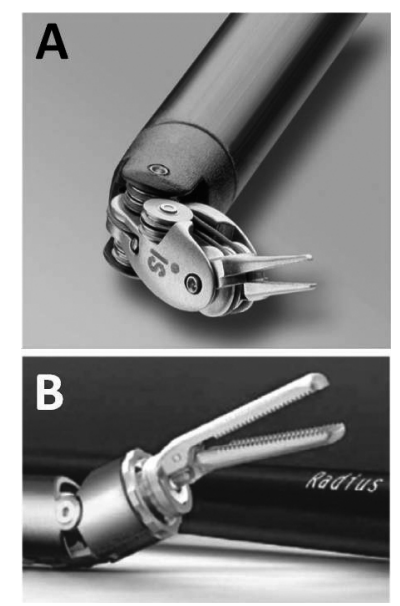

Figure 2. da Vinci's instrument tips (A) have complete freedom and articulation rather than the Radius' $(B)$.
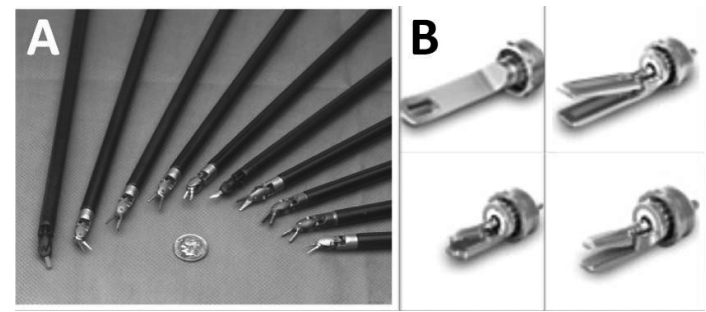

Figure 3. da Vinci has more than 40 instrument tips (A); Radius has only 4 tips (B). ments of the surgeon's hand, wrist and fingers into precise, real-time movements of the surgical instruments. The system can be downscaled by adjusting the ratio of motion of the handles to that of the surgical instruments to $2: 1,3: 1$ or $5: 1$. Another feature of the system is a motion filter, which removes unintended movements caused by human tremor. These technical advantages allow the surgeon to perform microsutures with relative ease and with high precision in a deep operative field through a small incision even when the suture is as thin as 8-0. In contrast, the Radius is manipulated through the port axis, which inevitably causes tremor at the tip end due to the principle of leverage, making the performance of delicate maneuvers difficult. When compared with the usual endoscopic instruments, however, it provides greater dexterity, allowing safer and easier surgical manipulations. From the point of dexterity, still da Vinci has the advantage, and we think Radius is preferable for endoscopic suturing with under 4-0 thread rather than for other endoscopic procedures.

\subsection{Size}

Size is the only major disadvantage of the da Vinci system. Its bulk makes access to the patient a little difficult. In robotic surgeries, the ideal positioning of the system, the optimal working angles and the proper port placement should be taken into consideration. For usability, the Radius has the advantage in terms of its size and quicker set-up time. The diameters for the da Vinci and the Radius instruments are $8 \mathrm{~mm}$ and $5 \mathrm{~mm}$.

\subsection{Tactility}

When the da Vinci system is used, the operating surgeon at the surgeon's console cannot receive information about the consistency of the tissue and sutures. For this reason, the surgeon has to rely on the visual feedback obtained through the three chip camera. In contrast, the surgeon using Radius can receive tactile feedback similar to conventional endoscopic instruments. However, lack of tactile feeling is not considered a serious concern for those of us who perform occasional robotic surgeries, and most surgeons should know the consistency and the vulnerability of target tissue from the experience they have gained through the performance of normal surgeries.

\subsection{Learning Curve}

In comparing the learning curves for both systems, we formed the impression that the da Vinci curve is much shorter than that for the Radius. The da Vinci system is designed to allow the surgeon the natural hand-eye alignment at the surgeon's console for intuitive control of the instrument. On the other hand, the Radius requires a sig- 
nificant amount of practice to acquire the skill needed to manipulate the instrument with unique movements. That is, it takes longer to learn how to manipulate the Radius than to master conventional endoscopic instruments.

\subsection{Economic Viewpoint}

The greatest disadvantage of the da Vinci is the cost of the main system, and of its maintenance and consumables, such as instruments. In Japan, The entire system of da Vinci S HD Surgical System including the 3D optical system is estimated at roughly about $\$ 3,750,000$. In addition, the average cost of each instrument is estimated at roughly \$500 per case depending on the procedure. At the same time, the Radius system costs approximately $\$ 35,000$ and its interchangeable surgical endo-effectors only cost an average of $\$ 200$ per case.

\subsection{CE Mark, FDA Clearance and Medical Device Approval in Japan}

The Radius has both the European CE Mark and Medical Device Approval in Japan, but it has not been approved by the US Food and Drug Administration (FDA). The da Vinci has the European CE Mark and the approval for several procedures from the FDA. Because da Vinci has been approved by the MHLW (Ministry of Health, Labour and Welfare) for only radical prostatectomy in Japan in 2012, and, other da Vinci surgeries cannot be covered by National Health Insurance, which becomes the most critical issue when performing the robotic surgery. Under such a circumstance, da Vinci surgeries currently can only be performed either under private practice, where the patient pays for the procedure or for research purposes, where the hospital shoulders the costs for such a procedure.

\section{Discussion}

The Radius system was originally developed to fill in the space between conventional surgical instruments and surgical robotics. Having specifically focused on ligation and suturing with suture sizes bigger than 4-0, we believe that it is more effective, less expensive and requires no extra effort for preparation compared with the da Vinci $[3,8,9]$. It is possible that use of the Radius system for suturing is less likely to be associated with litigation provided that it is used by a surgeon who has acquired the necessary skill through sufficient practice. A further expectation is that its diameter will be smaller than the existing one.

Although the da Vinci system is bulky, it allows surgeons to perform endoscopic surgeries under magnified $3 \mathrm{D}$ vision with high resolution only if ports are properly placed to prevent each arm from colliding with the other arms.
A crucial difference between the Radius and the da Vinci is not limited to anastomose small vessels such as coronary arteries but is extended to multidirectional dissection [10]. Currently, the cost including initial investment is the biggest issue; however, the da Vinci is absolutely necessary to implement delicate cardiac surgeries endoscopically and less invasively. Early approval by the MHLW is urgently required in Japan.

Because the da Vinci is a master-slave telemanipulation system containing a 3D optical system with superior resolution, we have already developed a 3D monitor for routine endoscopic surgeries and have employed it in clinical cases $[11,12]$. It can be assumed that if Radius could be used with a 3D monitoring system, it would be more useful.

Although both the da Vinci and the Radius systems have endoscopic instruments with a multi-degree of freedom and difference of the da Vinci and Radius is like comparing a sports car to a bicycle and stating the comparison needs to be made because they are forms of transportation and they have wheels. However, every hospital cannot buy a "sports car" because of the economical problem, and the most important message of this paper to surgeons and hospitals is the capabilities and costs of each system. Of course da Vinci is an expensive surgical robot, and most of endoscopic procedures can be performed by the da Vinci. However there is the operation that even Radius systems can be performed useful enough in endoscopic surgery. Applications need to be differentiated depending on the procedures and indications, and surgeon can perform excellent operations economically by using Radius systems appropriately.

\section{REFERENCES}

[1] N. Ishikawa, G. Watanabe, K. Iino, S Tomita, S. Yamaguchi, H. Kamiya, K. Higashidani, K. Kawachi and N. Inaki, "Robotic Internal Thoracic Artery Harvesting," Surgery Today, Vol. 37, No. 11, 2007, pp. 944-946. doi:10.1007/s00595-007-3542-4

[2] N. Ishikawa, G. Watanabe, K. Iino, S. Tomita, H. Nagamine and S Yamaguchi, “Japan's First Robot-assisted Totally Endoscopic Mitral Valve Repair with a Novel Atrial Retractor,” Artificial Organs, Vol. 33, No. 10, 2009, pp. 864-866. doi:10.1111/j.1525-1594.2009.00822.x

[3] N. Ishikawa, M. Kawaguchi, S. Shimizu, A. Matsunoki, N. Inaki and G. Watanabe, "Single-Incision Laparoscopic Hernioplasty Collaborate with Radius Surgical System," Surgical Endoscopy, Vol. 24, No. 6, 2010, pp. 730-731. doi:10.1007/s00464-009-0633-X

[4] N. Ishikawa, G. Watanabe, Y. Hirano, I. Inaki, K. Kawachi and M. Oda, "Robotic Dexterity: Evaluation of Three-Dimensional Monitoring System and Non-Dominant Hand Maneuverability in Robotic Surgery,” Journal of Robotic Surgery, Vol. 1, No. 3, 2007, pp. 231-233. 


\section{doi:10.1007/s11701-007-0037-7}

[5] S. D. Heazrrell, D. M. Kwartowitz, P. M. Milhoua and R. L. Galloway, "Toward Image Guided Robotic Surgery: System Validation," The Journal of Urology, Vol. 181, No. 2, 2009, pp. 783-789. doi:10.1016/j.juro.2008.10.022

[6] S. Yoshida, I. Yoshino, Y. Moriya, H. Hoshino, T. Okamoto, M. Suzuki and K. Shibuya, "Video-Assisted Thoracoscopic Surgery Extended Thymectomy for Myasthenia Gravis Using Manual Manipulators: The Radius Surgical System,” The Annals of Thoracic Surgery, Vol. 92, No. 6, 2011, pp. 2246-2248. doi:10.1016/j.athoracsur.2011.05.031

[7] K. Shibao, A. Higure, K. Yamaguchi and N. Minagawa, "Enucleation of an Insulinoma of the Pancreas Using Reduced Port Surgery: Report of a Case,” Surgical Technology International, Vol. 1, 2012, pp. 101-105.

[8] J. Bonatti, J. D. Lee, N. Bonaros, T. Schachner and E. J. Lehr, "Robotic Totally Endoscopic Multivessel Coronary Artery Bypass Grafting: Procedure Development, Challenges, Results,” Innovations, Vol. 7, No. 1, 2012, pp. 3-8.

[9] S. Srivastava, S. Gadasalli, M. Agusala, R. Kolluru, R.
Barrera, S. Quismundo, V. Srivastava and U. SeshadriKreaden, "Robotically Assisted Beating Heart Totally Endoscopic Coronary Artery Bypass (TECAB). Is There a Future?” Innovations, Vol. 3, No. 2, 2008, pp. 52-58.

[10] K. Suda, Y. Ishida, Y. Kawamura, K. Inaba, S. Kanaya, S. Teramukai, S. Satoh and I. Uyama, "Robot-Assisted Thoracoscopic Lymphadenectomy Along the Left Recurrent Laryngeal Nerve for Esophageal Squamous Cell Carcinoma in the Prone Position: Technical Report and ShortTerm Outcomes,” World Journal of Surgery, Vol. 36, No. 7, 2012, pp. 1608-1616. doi:10.1007/s00268-012-1538-8

[11] N. Ishikawa, M. Oda, H. Yasumatsu and G. Watanabe, “Three-Dimensional Monitor in Endoscopic Surgery," Surgical Endoscopy, Vol. 18, No. 7, 2004, pp. 149-150. doi:10.1007/s00464-003-8264-0

[12] S. Tomita, G. Watanabe, S. Tabata and S. Nishida, "Total Endoscopic Beating-Heart Coronary Artery Bypass Grafting Using a New 3D Imaging System,” Innovations, Vol. 1, No. 5, 2006, pp. 243-246. 